\title{
Boundary-Induced Phase Transitions in Equilibrium and Non-Equilibrium Systems
}

\author{
Malte Henkel ${ }^{a, \S}$ and Gunter Schütz ${ }^{b, \S}$ \\ ${ }^{a}$ Département de Physique Théorique, Université de Genève \\ 24 quai Ernest Ansermet, CH - 1211 Genève 4, Switzerland \\ ${ }^{b}$ Department of Physics, Weizmann Institute of Science, \\ Rehovot 76100, Israel \\ UGVA-DPT 1993/07-826 \\ WIS-93/73/Aug.-PH
}

\begin{abstract}
Boundary conditions may change the phase diagram of non-equilibrium statistical systems like the one-dimensional asymmetric simple exclusion process with and without particle number conservation. Using the quantum Hamiltonian approach, the model is mapped onto an XXZ quantum chain and solved using the Bethe ansatz. This system is related to a two-dimensional vertex model in thermal equilibrium. The phase transition caused by a point-like boundary defect in the dynamics of the one-dimensional exclusion model is in the same universality class as a continous (bulk) phase transition of the two-dimensional vertex model caused by a line defect at its boundary.
\end{abstract}

$\S$ Address after 1 October 1993: Department of Theoretical Physics, University of Oxford, 1 Keble Road, Oxford OX1 3NP, UK 
It is generally held that for statistical systems the influence of boundary conditions should be negligible, at least when one is interested in the bulk system only. This belief is indeed supported for equilibrium systems by abundant evidence coming from analytical or numerical studies. As we are going to show in this letter, however, this picture is far from being generally valid. Boundary conditions may indeed be crucial for the bulk properties and may even cause continous phase transitions.

It has already been realized that a change in boundary conditions (equivalent to some localized point defect in a system on a ring) may cause various kinds of phase transitions in the static properties of one-dimensional non-equilibrium systems [1, 2]. Here we show that boundary terms may also induce phase transitions in the dynamics of these systems. Such phase transitions are then shown to correspond to bulk phase transitions of two-dimensional equilibrium systems caused by boundary terms (i.e., line defects).

The paradigmatic example we are going to study is the asymmetric simple exclusion process, see [3]. In its simplest version, without particle creation or annihilation, it is described by particles of a single species $A$ moving on a lattice. A given site $j$ can be occupied or empty at an instant of time $t$. A particle at site $j$ for time $t$ may hop at time $t+1$ to its right neighbor with rate $(1+\epsilon) / 2$ and to its left neighbor with rate $(1-\epsilon) / 2$, if the final site is empty. This simple model appears in a large variety of contexts. It has been argued to be in the same universality class as the noisy Burger's equation $\mid$ | This in turn is a one-dimensional version of the incompressible NavierStokes equation or else can be regarded as the one-dimensional Kardar-Zhang-Parisi equation describing the shape fluctuation in various growth models.

Using a master equation approach, the probability distribution function $P(\{\beta\} ; t)$ is obtained by solving $\partial_{t} P=-H P$, where $\beta(t)$ is a configuration of occupied and empty sites and the quantum Hamiltonian reads [曲]

$$
H=-\frac{1}{4} \sum_{j=1}^{L}\left[\vec{\sigma}_{j} \cdot \vec{\sigma}_{j+1}-1+i \epsilon\left(\sigma_{j}^{x} \sigma_{j+1}^{y}-\sigma_{j}^{y} \sigma_{j+1}^{x}\right)\right]
$$

where $L$ is the number of sites and $\sigma^{x, y, z}$ are Pauli matrices. The ground state energy 
vanishes as follows from probability conservation in the master equation. Obviously $H$ commutes with the particle number operator $N=\sum_{j=1}^{L} n_{j}$ where we have introduced the particle projector $n_{j}=\frac{1}{2}\left(1-\sigma_{j}^{z}\right)$. The particle density is then $\rho=N / L$. Using periodic boundary conditions $\sigma_{L+1}^{x, y, z}=\sigma_{1}^{x, y, z}$, Gwa and Spohn [4] then show using Bethe ansatz techniques for $\epsilon=1$ and $\rho=1 / 2$ that the (real part of the) energies $E_{L}$ for $L$ large scale as

$$
E_{L} \sim L^{-3 / 2}
$$

On the other hand, the Hamiltonian had also been derived considering asymmetric lattice diffusion for the case of free boundary conditions where the particles are not allowed to move beyond the boundary (impenetrable walls). Then it reads [5]

$$
H^{\prime}=-\frac{1}{4 \Delta} \sum_{j=1}^{L-1}\left[\sigma_{j}^{x} \sigma_{j+1}^{x}+\sigma_{j}^{y} \sigma_{j+1}^{y}+\Delta \sigma_{j}^{z} \sigma_{j+1}^{z}-\frac{q-q^{-1}}{2}\left(\sigma_{j}^{z}-\sigma_{j+1}^{z}\right)-\Delta\right]
$$

where

$$
\Delta=\frac{q+q^{-1}}{2}, q=\sqrt{\frac{1-\epsilon}{1+\epsilon}}
$$

This is the well-known XXZ quantum chain which is symmetric under the quantum group $U_{q} S U(2)$ as shown by Pasquier and Saleur [6]. We stress that the quantum Hamiltonian of the diffusion process on a periodic lattice cannot be obtained by simply taking periodic boundary conditions in eq. (3). Using the $U_{q} S U(2)$ symmetry it is easy to show that for $\epsilon \neq 0$ the energies for $L$ large become

$$
E_{L}^{\prime} \sim 1-\Delta^{-1}
$$

in each sector with $N$ particles, see [6, ], 8].

The apparent inconsistency of eqs. (2, 5) will be explained below. Our analysis applies to any value of $\epsilon$, generalising the approach of 4 . We note that since the Hamiltonian of eq. (11) is gapless, the approach of time-dependent mean values towards their equilibrium value may be according to a power law, while with the Hamiltonian of eq. (函) the relaxation will be always exponential. 
To understand this observation, we begin by rewriting eq. (11). Introducing raising and lowering operators $\sigma^{ \pm}=\frac{1}{2}\left(\sigma^{x} \pm i \sigma^{y}\right)$, we have

$$
\begin{aligned}
H & =-\frac{1+\epsilon}{2} \sum_{j=1}^{L}\left[\frac{1-\epsilon}{1+\epsilon} \sigma_{j}^{+} \sigma_{j+1}^{-}+\sigma_{j}^{-} \sigma_{j+1}^{+}+\frac{1}{2(1+\epsilon)}\left(\sigma_{j}^{z} \sigma_{j+1}^{z}-1\right)\right] \\
& =-\frac{1}{q+q^{-1}} \sum_{j=1}^{L}\left[q \sigma_{j}^{+} \sigma_{j+1}^{-}+q^{-1} \sigma_{j}^{-} \sigma_{j+1}^{+}+\frac{q+q^{-1}}{4}\left(\sigma_{j}^{z} \sigma_{j+1}^{z}-1\right)\right]
\end{aligned}
$$

where the second of the eqs. (4) has been used. Next, consider the non-singular operator

$$
U=\exp \left(\pi g \sum_{j=1}^{L} j \sigma_{j}^{z}\right), U \sigma_{j}^{ \pm} U^{-1}=e^{ \pm 2 \pi g j} \sigma_{j}^{ \pm}
$$

If we now choose $q=e^{2 \pi g}$, we obtain

$$
H^{\prime \prime}=U H U^{-1}=-\frac{1}{2\left(q+q^{-1}\right)} \sum_{j=1}^{L}\left[\sigma_{j}^{x} \sigma_{j+1}^{x}+\sigma_{j}^{y} \sigma_{j+1}^{y}+\Delta \sigma_{j}^{z} \sigma_{j+1}^{z}-\Delta\right]
$$

which is indeed (almost) the Hamiltonian $H^{\prime}$. The distinction comes from the boundary conditions. The surface field $\left(q-q^{-1}\right)\left(\sigma_{1}^{z}-\sigma_{L}^{z}\right) / 8 \Delta$ is absent in $H^{\prime \prime}$ and one has

$$
\sigma_{L+1}^{ \pm}=q^{\mp L} \sigma_{1}^{ \pm}, \quad \sigma_{L+1}^{z}=\sigma_{1}^{z}
$$

which make $H^{\prime \prime}$ non-hermitian, as $H$ already is. It is these unusal boundary conditions which give rise to the different properties of $H$ and $H^{\prime}$, as we now show.

First we note that the ground state of the system (3) with free boundary conditions as well as that of the model (8) with twisted boundary conditions (9) is $(L+1)$-times degenerate and has energy 0 , independent of $L$. In what follows, we assume $L$ to be even. In each sector with fixed particle number $N$ the lowest energy is 0 . This can be shown by either solving the Bethe ansatz equations or by using the symmetry relations with the generators of the quantum algebra [6, 8, 9].

The calculation of the spectrum proceeds via the Bethe ansatz. Indeed, the XXZ chain with non-periodic boundary conditions was already considered in [10] and we merely have to adapt their results to the problem at hand. To begin with, we consider the sector with $N=1$ particle. Then the energies are in this sector

$$
E=1-\Delta^{-1} \cos \theta, \quad \theta=2 \pi\left(i g+\frac{n}{L}\right)
$$


where $n$ is an integer from the set $\{0, \pm 1, \ldots, \pm(L / 2-1), L / 2\}$. For $L$ large, the energies become

$$
\begin{aligned}
E & =1-\frac{\cos \left(2 \pi\left(i g+\frac{n}{L}\right)\right)}{\cosh (2 \pi g)} \\
& \simeq 2 \pi^{2}\left(\frac{n}{L}\right)^{2}+2 \pi i \tanh (2 \pi g) \frac{n}{L}+\ldots
\end{aligned}
$$

and we note that the mass term indeed cancels. We also observe that the real part $\Re E \sim L^{-2}$ and the imaginary part $\Im E \sim L^{-1}$. We return to an explanation of this below.

Next, we take the sector $N=2$. From the Bethe ansatz [10] we have

$$
\begin{aligned}
& E=\left(2 \Delta-\cos \theta-\cos \theta^{\prime}\right) \Delta^{-1} \\
& \theta L-2 \pi i g L=2 \pi I-\Theta\left(\theta, \theta^{\prime}\right), \quad \theta^{\prime} L-2 \pi i g L=2 \pi I^{\prime}-\Theta\left(\theta^{\prime}, \theta\right)
\end{aligned}
$$

where $I, I^{\prime}$ are distinct half-integers from the set $\left\{ \pm \frac{1}{2}, \pm \frac{3}{2}, \ldots, \pm \frac{L-1}{2}\right\}$ and

$$
\Theta\left(\theta, \theta^{\prime}\right)=2 \arctan \left(\frac{\Delta \sin \left(\frac{1}{2}\left(\theta-\theta^{\prime}\right)\right)}{\cos \left(\frac{1}{2}\left(\theta+\theta^{\prime}\right)\right)-\Delta \cos \left(\frac{1}{2}\left(\theta-\theta^{\prime}\right)\right)}\right)
$$

Define $\widetilde{\theta}=\theta-2 \pi i g, \widetilde{\theta}^{\prime}=\theta^{\prime}-2 \pi i g$. Then, for small values of the arguments

$$
\Theta\left(\widetilde{\theta}, \widetilde{\theta^{\prime}}\right) \simeq 2 \arctan \left(i \operatorname{coth}(2 \pi g) \cdot \frac{\widetilde{\theta}-\widetilde{\theta^{\prime}}}{\widetilde{\theta}+\widetilde{\theta^{\prime}}}\right)
$$

which is of order unity. Therefore

$$
\widetilde{\theta}=\frac{2 \pi}{L} a, \quad \widetilde{\theta^{\prime}}=\frac{2 \pi}{L} a^{\prime}
$$

where $a, a^{\prime}$ are of order $\mathcal{O}(1)$. It follows that the same cancellation as observed in the sector $N=1$ also takes place here and also that the observed scaling of the energies does not change. Finally, for $N$ arbitrary

$$
E=\Delta^{-1}\left(N \Delta-\sum_{n=1}^{N} \cos \theta_{n}\right)
$$

with

$$
\theta_{m} L-2 \pi i g L=2 \pi I_{m}-\sum_{n=1}^{N} \Theta\left(\theta_{m}, \theta_{n}\right)
$$


and the same argument can be repeated. It follows that for any value of $\epsilon$, the spectrum is massless. There is a distinction, however, between finite particle densities $\rho=N / L=\mathcal{O}(1)$ and small densities $\rho=\mathcal{O}\left(L^{-1}\right)$. As shown above, in the latter case the real part of the energy gaps scales as $L^{-2}$, while for $\rho=1 / 2$ it vanishes like $L^{-3 / 2}$ (when $\epsilon=1$ ) [4]. The way how this result was achieved suggests that this scaling behaviour is characteristic for all finite densities.

After this analysis of the spectra of $H^{\prime}$ and $H^{\prime \prime}$ (or $H$, as the spectra of $H$ and $H^{\prime \prime}$ are identical) we turn to an interpretation of our results. The $N$-particle ground state $|N\rangle$ is stationary with respect to the stochastic process defined by the Hamiltonian and we shall refer to it as the steady state of the system. Average values $\langle C\rangle$ of operators $C$ in some $N$-particle probability distribution $\left|P_{N}\right\rangle$ are defined as $\langle C\rangle=$ $\left\langle N|C| P_{N}\right\rangle$. Of particular interest are the $n$-point correlation functions in the steady state

$$
G\left(x_{n}, t_{n} ; \ldots ; x_{1}, t_{1} ; 0,0\right)=\left\langle N\left|n_{x_{n}} \mathrm{e}^{-H\left(t_{n}-t_{n-1}\right)} \ldots n_{x_{1}} \mathrm{e}^{-H t_{1}} n_{0}\right| N\right\rangle
$$

and their connected counterparts $G_{c}$ defined by replacing the operators $n_{x}$ by $\left(n_{x}-\rho\right)$ in $(18)$.

We discuss the system dynamics by studying the dynamic structure function $S_{N}(k, t)$ which is the Fourier transform of the connected two-point correlation function in the $N$-particle sector. In the case of asymmetric diffusion with free boundary conditions (3) the correlation function decays exponentially in time with finite relaxation time $\tau=\Delta /(\Delta-1)$, see eq. (5).

$$
S_{N}(k, t) \propto f(k) \mathrm{e}^{-t / \tau}
$$

The relaxation time does not depend on the density $\rho$. In the first model, however, corresponding to asymmetric diffusion with periodic boundary conditions, the relaxation time diverges. For small densities (finite number of particles) we read from eq. (11) $\tau \sim L^{2}$ while the result obtained by Gwa and Spohn translates into $\tau \sim L^{3 / 2}$ for finite densities. For the dynamic structure function this suggests the scaling form 
[4]

$$
S(k, t) \propto \mathrm{e}^{-i \epsilon(1-2 \rho) k t} h\left(k^{3 / 2} t\right)
$$

for finite densities while computing the exact structure function for one particle from eq. (10) suggests

$$
S(k, t) \propto \mathrm{e}^{-i \epsilon k t} \mathrm{e}^{-\frac{1}{2} k^{2} t}
$$

for any finite number of particles. The phase factors exp $(-i \epsilon(1-2 \rho) t)$ arise from the imaginary part of the energy gaps and reflect the steady state current of particles moving around the ring (recall $\tanh (2 \pi g)=-\epsilon)$. The scaling functions $h\left(k^{3 / 2} t\right)$ and $\exp \left(-k^{2} t / 2\right)$ have their origin in the diffusive nature of the process. From these expressions one can read the dynamic exponent of the process: it is $z=2$ for small densities and $z=3 / 2$ for finite densities. It is interesting to note the consistency with the results for the diffusion constant $D$ of a single tagged particle in the fully asymmetric exclusion model studied by Derrida et al. [11. These authors consider an exclusion model of the kind discussed here containing one particle which has the same dynamics as all the other (indistinguishable) particles, but is tagged. (Effectively, this a model with two types of particles, $\mathrm{A}$ and $\mathrm{B}$, containing $N_{A}$ particles of type $\mathrm{A}$ and $N_{B}=1$ particle of type B all moving with the same probability to the right if the neighbouring site was empty.) They follow the motion of the tagged particle and compute its diffusion constant $D$. They find that $D$ is finite for a finite number of untagged particles, corresponding to an dynamical exponent $z=2$. On the other hand $D$ diverges proportional to $L^{1 / 2}$ for finite densities which corresponds to $z=3 / 2$.

The discussion of behaviour of the dynamic structure function for free boundary conditions (19) on the one hand and for twisted (periodic) boundary conditions (20) and (21) on the other hand shows that a phase transition takes place when changing the boundary conditions of the model and elucidates its effect on the dynamics of the system. Now we show that this corresponds to a bulk phase transition of a twodimensional six-vertex model. The Hamiltonians $H_{L}^{\prime}$ and $H_{L}^{\prime \prime}$ of the model with $L$ sites can be derived from the transfer matrix $T_{L}$ of the six-vertex model with a defect line 
of the type given in eq. (9) located between sites $L$ and 1 (which we call the boundary) 24, 4. Its partition function $Z$ in thermal equilibrium on a $L \times M$ lattice is given by the trace of the $M^{t h}$ power of $T_{L}$. In the thermodynamic limit $L, M \rightarrow \infty$ one obtains $Z=\lim _{L, M \rightarrow \infty} \operatorname{Tr}\left[\exp \left(-H_{L} M\right)\right]$. From the vanishing of the energy gaps discussed above (which corresponds to the appearance of an infinite degeneracy of the ground state of $H$ ) we conclude that changing the boundary conditions causes a continous phase transition in the two-dimensional six-vertex model in thermal equilibrium.

So far we have studied asymmetric diffusion with a conserved number of particles. We briefly show that a boundary induced phase transition occurs also in an asymmetric diffusion model with pair annihilation $A+A \rightarrow \emptyset$. We study the Hamiltonian

$$
H=-\frac{1}{2\left(q+q^{-1}\right)}\left(\sum_{j=1}^{L-1} h_{j}+b h_{L}\right)
$$

with

$$
h_{j}=q \sigma_{j}^{+} \sigma_{j+1}^{-}+q^{-1} \sigma_{j}^{-} \sigma_{j+1}^{+}+\frac{q+q^{-1}}{2}\left(\sigma_{j}^{+} \sigma_{j+1}^{+}-2\right)+q \sigma_{j}^{z}+q^{-1} \sigma_{j+1}^{z} .
$$

The boundary conditions are defined by the parameter $b$. If $b=0$ one has free boundary conditions (we call this Hamiltonian $H^{F}$ when a distinction w.r.t. the boundary conditions is necessary) while $b=1$ correspond to periodic boundary conditions (denoted $H^{P}$ ). $H$ describes a process where particles in a pair of sites hop with probability $(1+\epsilon) / 2$ to the right and probability $(1-\epsilon) / 2$ to the left if the respective sites are empty. Pairs of particles are always annihilated. $H$ has a twofold degenerate steady state with energy 0 . These are the ferromagnetic ground state containing no particles (all spins up) and the one-particle state (one spin down) in which each possible position of the particle is equally probable.

The operator $U$ eq. ( (7) transforms $H^{F}$ into the free fermion Hamiltonian studied in [5] which describes a transition of the Pokrovsky-Talapov type [12]. The energy gaps are all finite and of the form

$$
E^{F} \sim N\left(1-\frac{2}{q+q^{-1}}\right)
$$


for large $L$, which implies an exponentially slow approach to the stationary state at late times.

For periodic boundary conditions $H^{P}$ is transformed by $U$ into

$$
H^{P^{\prime}}=-\frac{1}{2\left(q+q^{-1}\right)} \sum_{j=1}^{L}\left[\sigma_{j}^{x} \sigma_{j+1}^{x}+\sigma_{j}^{y} \sigma_{j+1}^{y}+\frac{q+q^{-1}}{2}\left(q^{2 j+1} \sigma_{j}^{+} \sigma_{j+1}^{+}-2\right)+q \sigma_{j}^{z}+q^{-1} \sigma_{j+1}^{z}\right]
$$

with the twisted boundary conditions eq. (9). Following the argument of [5], the spectrum of $H^{P^{\prime}}$ is seen to be independent of the annihilation term $\sigma_{j}^{+} \sigma_{j+1}^{+}$and it is therefore equal to the spectrum of

$$
H_{0}^{P^{\prime}}=-\frac{1}{2\left(q+q^{-1}\right)} \sum_{j=1}^{L}\left[\sigma_{j}^{x} \sigma_{j+1}^{x}+\sigma_{j}^{y} \sigma_{j+1}^{y}+\frac{q+q^{-1}}{2}\left(\sigma_{j}^{z}+\sigma_{j+1}^{z}-2\right)\right]
$$

which commutes with the particle number operator $N$ and describes non-interacting fermions on a ring with twisted boundary conditions eq. (9). The eigenvalues of $H_{0}^{P^{\prime}}$ are easily found by a Jordan-Wigner and Fourier transformation and are of the form

$$
E^{P}=\sum_{i=1}^{N}\left(1-\frac{2}{q+q^{-1}} \cos \theta_{i}\right)
$$

similar to eq. (16) but with

$$
\theta_{i}=2 \pi\left(i g+m_{i} / L\right)
$$

where the $m_{i}$ are pairwise distinct integers $0 \leq m_{i} \leq L-1$ for $N$ odd and halfintegers $\frac{1}{2} \leq m_{i} \leq L-\frac{1}{2}$ for $N$ even. The low lying energy gaps ( $N$ finite) vanish in the thermodynamic limit as $\Re E^{P} \sim L^{-2}$ and $\Im E^{P} \sim L^{-1}$. Here, as opposed to the model with free boundary conditions, finite-density states decay with a relaxation time of order 1 , while for low-density states (finite $N$ ) the relaxation time diverges proportional to $L^{2}$.

The Hamiltonian (22) is related to a 7-vertex model with a boundary defect, see [5]. As in the 6-vertex model discussed above, changing the boundary condition induces a bulk phase transition. The same mechanism should also apply to more general reaction-diffusion problems, for example two-particle processes with a reaction $A+B \rightarrow \emptyset$ [13, or even extensions of the Hubbard model [14, where the same quantum chains as considered here reappear [5]. 
To summarize, we have shown that boundary conditions can have a major influence on the phase diagram of certain statistical models. Since the models considered here are merely prototypes of much more general ones, we expect that the phenomenon found is generic.

\section{Acknowledgements}

M.H. thanks the Swiss National Science Foundation for support. G.S. would like to thank H. Spohn for useful discussions. Financial support by the Deutsche Forschungsgemeinschaft is gratefully acknowledged. 


\section{References}

[1] J. Krug, Phys. Rev. Lett. 67, 1882 (1991); S.A. Janowsky and J.L. Lebowitz, Phys. Rev. A45, 618 (1992); D. Kandel and D. Mukamel, Europhys. Lett. 20, 325 (1992); B. Derrida, E. Domany and D. Mukamel, J. Stat. Phys. 69, 667 (1992); G. Schütz and E. Domany, J. Stat. Phys. 72 (in press)

[2] G. Schütz, J. Stat. Phys. 71, 471 (1993); Phys. Rev. E47, 4265 (1993)

[3] T. Ligget, Interacting Particle Systems, Springer, Berlin (1985)

[4] L.-H. Gwa and H. Spohn, Phys. Rev. A46, 844 (1992)

[5] F.C. Alcaraz, M. Droz, M. Henkel and V. Rittenberg, Ann. of Phys., to appear

[6] V. Pasquier and H. Saleur, Nucl. Phys. B330, 523 (1990)

[7] J.D. Johnson and B.M. McCoy, Phys. Rev. A6, 1613 (1972); M. Takanishi, Prog. Theor. Phys. 50, 1519 (1973); 51, 1348 (1974)

[8] S. Sandow and G. Schütz, Weizmann preprint (1993)

[9] U. Grimm and G. Schütz, J. Stat. Phys. 71, 927 (1993)

[10] F.C. Alcaraz, M.N. Barber and M.T. Batchelor, Ann. of Phys. 182, 280 (1988)

[11] B. Derrida, M.R. Evans and D. Mukamel, Saclay preprint (1993)

[12] V.L. Pokrovsky and A.L. Talapov, Sov. Phys. JETP 51, 134 (1980)

[13] V. Privman, Phys. Rev. A46, R6140 (1992); F.C. Alcaraz and V. Rittenberg, Bonn preprint (1993)

[14] F.C. Alcaraz, D. Arnaudon, V. Rittenberg and M. Scheunert, CERN preprint TH.6935/93 